\title{
EL TURISMO RURAL COMUNITARIO ¿UNA OPORTUNIDAD EN EL POSCONFLICTO COLOMBIANO?
}

\author{
JORGE ALEXANDER MORA FORERO ${ }^{1}$ \\ Docente y Líder Investigador \\ Programa Administración de Empresas Turísticas y Hoteleras \\ UNIMINUTO Virtual y a Distancia \\ Bogotá - Colombia \\ LUIS MAURICIO BOHORQUEZ PATIÑO \\ Estudiante semillero Tornare \\ Programa Administración de Empresas Turísticas y Hoteleras \\ UNIMINUTO Virtual y a Distancia \\ Bogotá - Colombia
}

\section{Resumen}

La actividad turística en Colombia es una práctica incipiente, no obstante, ha obtenido un papel muy importante en la economía del país. Incluso, en la actualidad el gobierno central está haciendo esfuerzos para posicionar al turismo como una actividad económica sólida. Este gran momento del turismo en el país, ha hecho que muchos turistas busquen experiencias que anteriormente no eran tenidas en cuenta. Es allí donde el turismo comunitario se ha impuesto como una tendencia turística que es importante analizar. Por ello, esta investigación intenta responder la relación de esta forma de turismo y el aporte que ha tenido para el desarrollo rural de la población local. Para ello, esta investigaciónrealizo un estudio de caso el Páramo de Sumapaz,ubicado en la ciudad de Bogotá D.C., en la localidad con su mismo nombre. Este lugar fue el epicentro por muchos años del conflicto interno del país.Allí se realizó un análisis con la comunidad acerca de las iniciativas turísticas y su importancia para el desarrollo económico de la comunidad.En este territorio en los últimos años se ha convertido en un atractivo turístico, dado que es un lugar de posconflicto. Incluso, algunos pobladores han visto en el turismo una alternativa de ingresos económicos adicionales. Sin embargo, se evidencia una serie de retos debido a que hay problemáticas que amenazan a la comunidad, los turistas y al medio ambiente. Sin embargo, con una buena gestión se puede lograr un turismo responsable y sostenible.

Palabras Clave: Turismocomunitario, posconflicto, desarrollo rural

\section{Abstract}

The tourist activity in Colombia is an incipient practice, nevertheless, it has obtained a very important role in the economy of the country. Even today, the central government is making efforts to position tourism as a solid economic activity. This great moment of tourism in the country, has made many tourists seek experiences that were not previouslytaken into

\footnotetext{
1jorge.moraf@uniminuto.edu
} 
account. This is where community based tourism has become a tourist trend that is important to analyze. Therefore, this research tries to answer the relationship of this form of tourism and the contribution it has made to the rural development of the local population. To this end, this research carried out a case of study at the Paramo de Sumapaz, located in the city of Bogotá D.C., in the town with the same name. This place was the epicenter for many years of the internal conflict of the country. There, an analysis was made with the community about tourism initiatives and their importance for the economic development of the community. This territory in recent years has become a tourist attraction, given that it is a post-conflict place and some residents have found in tourism an alternative of additional economic income. However, a series of challenges is evident due to the fact that there are problems that threaten the community, tourists and the environment. Nonetheless, with a good management, responsible and sustainable tourism can be achieved.

Key Words: Rural development, community based tourism, post-conflict

\section{1-INTRODUCCIÓN}

Luego de la firmade los acuerdos de paz en Colombia en el año 2016, la actividad turística fue una de las grandes favorecidas (Esteban \& Bonilla, 2017).El turismo por muchos años no tuvo un desarrollo significativo, debido al conflicto interno, lo cual ha venido afecto en mayores proporciones a las comunidades rurales. Esto ha entorpecido el desarrollo óptimo de aquellas actividades propias de las poblaciones rurales como lo son la agricultura, la ganadería y demás labores campesinas. No obstante, el turismo surge ante esta circunstancia como una oportunidad de desarrollo económico para las comunidades rurales campesinas.

Por más de cinco décadas, Colombia enfrentó un conflicto interno que perjudicó a todos sus habitantes, pero en especial a todos aquellos que vivían en territorios rurales debido a que por las complejas condiciones del territorio colombiano, sumado a la poca presencia del estado en dichas áreas, se convierten en circunstancias favorables para aquellos grupos armados ilegales cometieran sus crímenes. Por esto el conflicto tuvo un mayor impacto en la población campesina y esto afecto algunas de sus doctrinas sociales y económicas, como lo son la producción agrícola y ganadera.(Ibáñez \& Rodriguez, 2014). Entre los años 1999 y 2013, el conflicto armado ocasionó el desplazamiento de 3.9 millones de colombianos, entre los cuales el 55\% trabajaban y/o poseían tierras productoras en labores agrícolas y pecuarias(Ibáñez \& Rodriguez, 2014). Por otra parte, las diversas circunstancias han ocasionado cambios en el territorio y en algunos casos, las comunidades que habitan dichas zonas se han convertido en parte de un atractivo turístico. Debido a esto los locales han empezado a utilizar sus recursos adaptados al turismo, con el fin de obtener un beneficio e ingreso adicional que contribuya con su desarrollo (Guilland\& Ojeda, 2012).

Antes de abordar las concepciones del turismo es importante entender que este es un hecho ligado a una sociedad. Incluso, cuando se vea como un factor multidisciplinar, también se ve como una empresa y por esto se debe hablar de la gestión local y las pequeñas iniciativas; las cuales son las características de lo que se conoce como turismo comunitario. Teniendo en cuenta que este intenta ser una actividad sostenible, con respeto por cada uno de los sistemas que allí intervienen. De igual forma, el turismo comunitario se realiza como una empresa, generando oportunidades y en el caso de esta investigación se intenta analizar su relación con el desarrollo rural y la memoria de lo que se conoce como posconflicto en el 
país. También el turismo comunitario intenta dar una preservación y protección del entorno, con valores de un humanismo moderno.

El pensamiento social de este turismo plantea nuevas formas de pensamiento y reflexiones humanistas. El humanismo moderno propone que la "construcción del ser" es una intensión común y que allí habita la importancia de otro aspecto fundamental del pensamiento humanista,que plantea la "construcción de sí mismo" (Bokova, 2010). En esta pretensión colectiva permanece la importancia de otro aspecto fundamental del mensaje humanista, que hace se enfoca en la necesaria dimensión colectiva de toda vida humana consumada (Bokova, 2010). Siguiendo con este concepto, las personas solo pueden realizarse completamente si son parte de una comunidad; debido a esto se plantea el concepto de común unidad de seres humanos, la cual vincula a las personas entre sí. Mediante la diversidad es posible alcanzar una cultura humana en común que se ve reflejada a través de la comunicación, el aprendizaje y los dialectos.

\section{2- ESTADO DEL ARTE}

El turismo es un concepto complejo a la hora de dar una definición,Las definiciones que proponen diferentes autores hacen que se dé un debate epistémico bastante interesante. Dado que esta actividad es objeto de estudio de diferentes disciplinas; y los abordajes científicos del turismo son dados por lo general de acuerdo a la formación disciplinar de cada uno de los distintos autores. Del mismo modo sucede con el turismo comunitario, esta actividad tiene en cuenta diferentes elementos como se evidenciará a continuación.

\section{3- TURISMO COMUNITARIO}

El turismo comunitario surgió a finales del siglo XX y las primeras investigaciones que se conocieron pueden encontrarse comoCommunityBasedTourism (CBT).Esta alternativa para realizar actividades turísticas se remonta al año de 1970 e inicia como una alternativa de turismo de masas que se logró mediante agencias de viajes (Suriya, 2010). El turismo comunitario (CBT) por sus siglas en inglés, generalmente es asociado al turismo alternativo, caracterizándose por incluir elementos cuyo fin sea fomentar la preservación del entorno natural que se aprecia mediante demostraciones socioculturales de las comunidades (Domínguez, Bernard \& Burguete, 2018).

En el año de 1985 Murphy toma como objeto de estudio el turismo comunitario mediante su investigación, con el fin de exponer la relación que se puede dar entre la actividad turística y las comunidades locales apuntándole al desarrollo (Guzmán \& Cañizares, 2009).Dicha actividad está basada en técnicas de integración local con la comunidad, y para esto es indispensable lograr una conexión productiva que contribuya e incluya a las comunidades "fundamentado en la posesión y la auto administración de los recursos que son patrimonio de la comunidad, con aras de lograr un trabajo solidario y una distribución equitativa de los beneficios obtenidos con la actividad turística" (Maldonado, 2005).

Aunque el turismo comunitario se lleva a cabo en pequeñas proporciones, se establece como una alternativa adicional de ingresos en una comunidad, y para que esto sea posible debeexistir una relación entre diversos actores de la economía (Guzmán, Garduño \&Zizumbo, 2009).Dicha relación debe constituirse mediante actividades en las cuales todos 
los organismos de la comunidad logren una interacción entre sí. El turismo comunitario es una herramienta para preservar el medio ambiente e incrementar el desarrollo social y económico, cuando se realiza de manera sostenible; cuando es ejecutado y administrado por la misma comunidad en aras de combatir la pobreza.(Vargas, Ocaña \&Chaviano, 2017).

El CBT es un eje complementario que contribuye y sirve a la economía de la comunidad, la pluralidad económica, y es un elemento importante para la preservación del territorio local. Su ejecución tiene como principio el autoempleo y la obtención de utilidades tangibles e intangibles para la comunidad (Ruiz et al., 2008).La intervención activa de la comunidad y la constitución de sociedades con distintas actividades económicas, son la base del turismo comunitario, así como también lo es el fortalecimiento de las relaciones entre los turistas y los locales. (Guzmán \& Cañizares, 2009).

Carpetiere citado por Roux expresa que el turismo comunitario surge como una oportunidad de desarrollo para las comunidades locales, que se encontraban inquietas por cuidar de sus recursos naturales, culturales, sociales y de patrimonio, resistiendo la aparición de compañías dedicadas a la explotación y a los operadores turísticos privados (2013). El CBT no solo es una transfiguración para las comunidades locales, sino que también es una manera de fortalecerlas, integrarlas y suministrarles mayores recursos para que puedan tener una mayor autonomía en la administración y uso del territorio. El turismo comunitario más allá de ser una opción de metamorfosis de las comunidades, es una forma de consolidar a las comunidades y proporcionarles mayores recursos; de tal manera puedan gozar de mayor autonomía en la gestión del territorio (Ruiz, et al., 2008).

Actualmente el turismo basado en la comunidad se entiende como una estrategia de desarrollo en el ámbito cultural, social y económico, ya que se compone de una serie de prácticas tradicionales de dominio (Ballesteros, \&Carrion, 2007). Esta práctica busca desarrollar actividades turísticas en ambientes específicos, en los cuales las comunidades receptoras sean quienes autogestionen el turismo, convirtiéndose en su eje principal y siendo las primeras beneficiadas (Orgaz, 2013).Para ello, el turismo comunitario debe incorporarse al proyecto de vida de los locales; y así lograr una interacción holística de una buena aceptación por parte de la comunidad (De La Torre, 2010).

La actividad turística comunitaria tiende a generar diversos choques entre las comunidades y algunos sectores productivos ya que, en materia de desarrollo territorial, los locales defienden el medio ambiente mientras que la empresa privada y el sector de productivo tienen un concepto más lucrativo y menos respetuoso del mismo (Skewes, Zuñiga\& Vera, 2015). Lo anterior propone que la actividad turística no siempre goza de aceptación unánime y que su ejecución debe estar enfocado a los propósitos sociales y entornos comunitarios de acuerdo con los principios instaurados.

En algunas comunidades existen parámetros constituidos para la ejecución de sus actividades económicas, que resaltan una serie de valores y principios con el fin de integrar iniciativas turísticas dentro de su diario vivir, en las cuales es indispensable incluir procesos enfocados al desarrollo sostenible. Se ha evidenciado, el turismo comunitario cuenta con algunas variables que le apuntan a la sostenibilidad; y una de las más importantes es al turismo sostenible o sustentable (Ballesteros \&Carrion, 2007). Incluso el turismo comunitario es visto como una administración y autogestión de los atractivos turísticos. Por ello, el CBT tiene como objeto mitigar el impacto negativo y conseguir un beneficio mayor 
en cada uno de los factores que intervienen como lo son: el aspecto ambiental, económico, social y (Vera, Paladines \&Velasco, 2017).

Para conseguir un nivel visible en materia de sostenibilidad, es necesario que las comunidades trabajen solidariamente en la planificación y gestión turística donde los ingresos sean invertidos en sus propias necesidades y las del entorno (Inostroza, 2018).Toda iniciativa comunitaria está en la obligación de practicar y promover el turismo sostenible. Entendiendo este último como la conciencia sobre las consecuencias presentes y futuras, en los ámbitos sociales, culturales, económicos y medioambientales, con el fin de satisfacer las necesidades de los turistas, la empresa privada, y el territorio de las comunidades locales (OMT, 2018).

Se puede evidenciar que el CBT más allá de ser un modo de viajar, es una oportunidad de enriquecer la experiencia del turista y darle un valor adicional. Se puede concluir que el turismo comunitario tiene en cuenta las actividades realizadas por los visitantes o turistas dentro de las comunidades urbanas o rurales, en las cuales sus habitantes adoptan la práctica turística como una actividad económica adicional o principal, como una alternativa de ingresos. Allí los residentes participan activamente en la prestación de servicios y venta de productos turísticos, utilizando sus recursos ambientales y culturales de manera sostenible. Estas actividades deben ser rentables, y la mayoría del dinero generado por los turistas debe ser invertido para el desarrollo de la comunidad. Lo anterior demanda una fusión en la cual todos los locales que oferten productos y servicios relacionados con el turismo, trabajen de la mano y de manera organizada para que sus actividades económicas se vean bien constituidas.

\section{1-Actividades del Desarrollo Rural}

Los trabajos del campo como la agricultura son propios del territorio rural, no obstante, aparece el concepto de multifuncionalidad agraria, que propone una diversidad de actividades socioeconómicas relacionadas con dicha actividad y especialidades agropecuarias (Loewy, 2016).Lo anterior sugiere un planteamiento que da un giro a la perspectiva rural, e incluye un concepto de interrelación económica y social (Marzo, Pedraja\&Vinzón, 2017).

Es posible llevar a cabo múltiples actividades artesanales, industriales, comerciales, mineras y de extracción entre otras incluyendo el turismo en el desarrollo rural (Mora \&Cerón, 2015). Pueden encontrarse distintas oportunidades de acción que pueden ser alternadas en el desarrollo rural con productos, servicios y trabajos reflejados en artesanías, industria, comercio, y turismo pero deben ser aprovechadas de manera sostenible(Pérez, 2001).Puede entenderse el desarrollo rural como un pensamiento complejo de administración del territorio, de evolución, cambio y crecimiento en el cual, se entrecruzan distintas dimensiones como la cultura de identidad, la dimensión económica, política, ambiental, social y cultural(Aghón, Alburquerque \& Cortés,2001; Massolo, 2006).

Esta pluralidad da respuesta a las exigencias y la colaboración de distintos interventores con el objetivo de lograr el bienestar y una mejor calidad de vida (Cárdenas \& Vallejo, 2016). Es definida por su variedad en cada uno de los diferentes aspectos y presenta tendencia de valor y revalorización del entorno a través de la capitalización de sus recursos y su potencial (Aghón, Alburquerque \& Cortés, 2001).Este desarrollo del territorio rural se extiende a las fronteras de "lo local", y supone una tolerancia territorial que puede lograr 
alcances regionales e incluso nacionales (Aghón, Alburquerque \& Cortés, 2001; Massolo, 2006).

El desarrollo rural como concepto en Colombia tuvo como propósito afrontar y sobrepasar la pobreza rural bajo una reforma agraria, mediante mejores ingresos para dicha población y una propuesta que trabajara estas problemáticas (Cárdenas \& Vallejo, 2016).Desde estos inicios, la historia del desarrollo rural en Colombia no ha tenido cambios significativos pero, se implementaron bastantes mecanismos como la implementación de monocultivos, la expansión del área e intensificación de las actividades agropecuarias, y la mecanización entre otros (Cárdenas \& Vallejo, 2016).Sin embargo, estas medidas no han sido tan convenientes debido a la existencia de monopolios en las actividades económicas que tienen que ver con el desarrollo rural.

La carencia de inserción de un enfoque territorial que esté unido al desarrollo rural, la inclusión social, la perspectiva de género y la monopolización de una o más actividades entre otros, es lo que genera falencias que son visibles y están presentes(Carrillo \& Useche, 2017). Por ello se deben proponer modelos en la nueva ruralidad para el desarrollo como herramienta para construir paz en Colombia, y verlo desde un eje transversal con enfoque territorial y de género es necesario para lograr óptimos resultados (Fonseca et al., 2017). El gobierno no ha dado respuesta ni solución a las necesidades de la población rural desde sus entidades públicas (Ibáñez, 2016). Lo anterior implica que un gran rezago en el sector rural que se ha convertido en una tradición centralista.

El vínculo que existe entre la actividad turística y el desarrollo rural es muy claro; una actividad económica que supone una fuente de ingresos adicionales. Esto permite mejorar la calidad de vida, el bienestar de la población rural y por ende, es necesario que sea incorporado como una opción de desarrollo local para el posconflicto. Usualmente el turismo considera el uso del territorio como elemento primordial de la actividad turística, y representa un desplazamiento territorial que genera un intercambio de culturas, en el cual se explotan los recursos naturales y socioculturales de la localidad que en algunos casos se extienden a la región para obtener retribuciones económicas (Laguna, 2006).

\section{2- Turismo y posconflicto}

De acuerdo con la teoría del espacio, la actividad turística necesariamente supone un desplazamiento y se evidencia en la tesis deLeiper (1990), el territorio en dicho trabajo entiende un lugar de procedencia y/o destino final al cual se trasladan los turistas. Sin embargo, algunos de estos traslados se hacen contra de la voluntad de la población, como por ejemplo aquellos casos en que se presentan desplazamientos forzosos. Este tipo de situaciones fatídicas para la población, generan interés en algunas personas y son vistos como consecuencias históricas que pueden convertirse en atractivos turísticosde valorización social (Almirón, 2004).

Lo anterior demuestra que el turismo tiene gran potencial en aquellos escenarios donde se han presentado conflictos armados, atentados en contra de la integridad humana y crímenes de lesa humanidad. Un ejemplo claro es el campo de concentración Sachsenhausen ubicado en Alemania el cual fue un escenario de asesinatos, sometimiento de centenares de víctimas de la segunda guerra mundial entre los años de 1936 y 1945.(Davis \& Bowring, 2011). Luego de dicha guerra, fué un espacio de reclusión y condenaban presos políticos en la época de la guerra fría. Este escenario se destacó por haber sido durante mucho tiempo un 
campo de concentración, exterminio y reclusión; actualmente ha sido convertido en un lugar de memoria visto como un atractivo turístico y sigue despertando curiosidad entre los visitantes.

Así como en Alemania, otros países como Ruanda y Camboya tuvieron acontecimientos que generaron como resultado monumentos de memoria en materia de posgenocidio, que de igual forma han atraído visitantes de todo el mundo (Davis \& Bowring, 2011). Dichos espacios fueron reconocidos en otro momento de la historia por sus guerras y conflictos; Actualmente son destinos turísticosque gozan de aceptación y alta afluencia de visitantes. Lo anterior es debido a la promoción que logró despertar la curiosidad e interés de los turistas en su mayoría extranjeros, mediante las excursiones y recorridos turísticos organizados en los cuales se exponen las historias de dichos acontecimientos. La actividad turística realizada en el periodo de posconflicto, procura que la sociedad cree alternativas de turismo mientras promueve la superación social de aquellas situaciones adversas generando memoria (Causevic y Lynch 2011). Este modelo turístico es visto como un proceso por el cual deben pasar los lugares antes de convertirse en atractivos turísticos para que la población entierre y adopten reconozcan como patrimonio su pasado (Esteban \& Bonilla, 2017). En Colombia, el concepto turismo y posconflicto es algo nuevo y está siendo expresado y aprovechado para promover la actividad turística y despertar interés en los visitantes tanto nacionales como regionales y del resto del mundo.

\section{4- MARCO METODOLÓGICO}

Esta investigación ha utilizado un enfoque de investigación cualitativo, y el tipo de investigación es descriptivo y pretende analizar la relación del turismo comunitario y como este se articula en la multifuncionalidad rural en la actual coyuntura del posconflicto colombiano. Para ello, se realizó un estudio de caso en una comunidad rural en el páramo más grande del mundo, el páramo del Sumapaz (Doria \& Cardona, 2015). Este lugar es un área protegida por el sistema de Parques Nacionales Naturales de Colombia (Sánchez, 2018).

Allí se realizó un dialogo con los líderes de la comunidad a través de entrevistas (Robertt\&Lisdero, 2016). Este dialogo con la comunidad pretende describir cual ha sido el avance del turismo después de la firma de los acuerdos a de Paz. También se intenta representar la relación que tuvo el Sumapaz con el conflicto. El trabajo de campo de esta investigación se desarrolló durante múltiples visitas a comienzos y mediados del año 2018.

\section{5- RESULTADOS}

La población local del Sumapaz indica que el territorio ha tenido una transformación constante, allí se ha evidenciado un incremento en los turistas. Incluso los líderes comunitarios afirman es utópico estimar la cantidad de turistas, debido a que la mayoría de turistas llegan por medio de agencia de viajes que no tienen relación con la comunidad. Además, muchos de los senderos a los cuales llegan los turistas no son áreas delimitadas para el turismo. Es decir, existe una ilegalidad en la mayoría de la actividad turísticas de este territorio.

De igual forma es importante hacer énfasis que hay algunas propuestas comunitarias en pequeña escala. No obstante, estas se quedan pequeñas ante la escala de turistas que llegan a esta región. Si, existe una importancia del turismo para el desarrollo económico y social de unas pocas familias de la comunidad. 
En términos generales se evidencia una gran preocupación teniendo en cuenta que son muchos los turistas y no hay un control al acceso. La comunidad expresa que no hay controles de los turistas y la mayoría de los turistas arriban al paramo más grande del mundo, lo hacen por medio de operadores turísticos que no tienen en cuenta a la población local; son pocas las comunidades que se han apropiado del turismo. La comunidad también expresa su preocupación, debido a que el turismo en este lugar no cuenta con un plan establecido y este puede ser una práctica destructora para el paisaje natural.

Además, de la amenaza que representan los turistas para el medio ambiente, se evidencia un peligro en doble vía. Debido a que según los relatos de la comunidad gran parte de este territorio son zonas que incluso inexploradas por la comunidad, debido a que aún hay zonas que se encuentran minadas y esto representa un peligro para los turistas. Incluso, en algunos lugares del páramo las caminatas ecológicas de índole turística, están restringidas por seguridad de los turistas y del territorio.

Es importante reconocer que a pesar de que esta zona fue un territorio estratégico para los grupos ilegales, no se evidencian historias del conflicto que tengan un potencial para generar un turismo de memoria. Se puede considerar que esta zona era estratégica para los grupos ilegales alzados en armas. Por lo tanto, no hay registros de grandes tragedias como en otras zonas del país.

\section{5- CONCLUSIONES}

A pesar de que el turismo aporta económicamente al desarrollo de la comunidad; su participación es muy baja. El turismo comunitario en este lugar se observa de forma incipiente y la gestión local se ve impedida por varias razones. En gran medida se debe a que la mayoría de los turistas que llegan a este lugar, lo hacen por medio de operadores que no tienen ninguna relación con la comunidad. El turismo comunitario como se mencionó anteriormente se caracteriza por la autogestión de los servicios turísticos; por parte de la comunidad receptora.

La zona del Sumapaz no se puede considerar un destino turístico comunitario, debido a que las iniciativas locales son escasas frente a las propuestas de las agencias de viajes que intervienen este territorio. Sin embargo, se observa una gran oportunidad para que la comunidad logre apropiarse del territorio y autogestionar el turismo. Para ello, se requiere de una concertación y buena voluntad por parte de la población local.

Es importante resaltar que el turismo es una actividad que debe tener un atractivo, por ello, las poblaciones locales pueden desarrollar iniciativas turísticas, sin descuidar las demás actividades económicas, como la agricultura. El turismo en la multifuncionalidad rural es una actividad que llega a ser un complemento o parte de una diversificación de la economía local.

Sin embargo, hay varias acciones que se deben mejorar. Entre ellas, que existan lineamientos claros para el turismo comunitarios. Más programas de apoyo para la planificación turística en las entidades territoriales.

Esta investigación tuvo en cuenta un estudio de caso y sus resultados y conclusiones deben ser comparadas con otros estudios en el país, con el fin de obtener una perspectiva más holística del turismo comunitario en Colombia. Esta aproximación permite reconocer las dinámicas sociales y económicas del turismo en el territorio rural. De igual forma se deben 
establecer estudios sociológicos y económicos del turismo en otras zonas del posconflicto, donde la violencia tuvo mayores impactos sociales, con el fin de determinar la relación del turismo de memoria.

Esta investigación puede servir como un eslabón para construir otros estudios que tengan relación con el turismo rural comunitario en la actual coyuntura de colombiana de posconflicto. No obstante, se deben dar estudios serios que evidencien las realidades de las otras zonas del país, debido a que esta investigación se queda corta, dados los múltiples escenarios de posconflicto que cuentan con algún potencial turístico comunitario.

\section{REFERENCIAS BIBLIOGRÁFICAS}

Aghón, G., Alburquerque, F. \& Cortés, P. (2001). Desarrollo económico local y descentralización en América Latina: Análisis comparativo. CEPAL/GTZ. Santiago de Chile.

Almirón, A. V. (2004). Turismo y espacio. Aportes para otra geografía del turismo. GEOUSP: Espaço e Tempo (Online), (16), 166-180.

Ballesteros, E. R., \&Carrion, D. S. (2007). Turismo comunitario en Ecuador: desarrollo y sostenibilidad social. Editorial Abya Yala

Bokova, I. (2010). Un nuevo Humanismo para el siglo XXI. Obtenido en http://unesdoc. unesco. org/images/0018/001897/189775s. pdf.

Cárdenas, J. \& Vallejo, L. (2016). Agricultura y Desarrollo Rural en Colombia 2011_2013: una aproximación. CENES, VOL. 35, No. 62. Pp. 87-123.

Carrillo Castelblanco, E. A., \& Useche Triana, D. (2017). Análisis del enfoque de nueva ruralidad como modelo de desarrollo e instrumento para la construcción de paz en Colombia.

Causevic, S., \& Lynch, P. (2011). Phoenix tourism: Post-conflict tourism role. Annals of Tourism Research, 38(3), 780-800.

Davis, S., \& Bowring, J. (2011). Connecting with tragedy through landscapes of memory: memorial design, tourism, and the post-genocide memoryscapes of Cambodia, Rwanda, and Germany. TheMemoryWaka .

De La Torre, S. (2010). Turismo comunitario, ¿otro sueño inalcanzable?.Polémika, 5(1).

Domínguez, P., Bernard, A., \& Burguete, E. (2018). Turismo Alternativo y Tecnología: Promoción de la Sierra Mágica por medio de internet. Gestión Turística, (2), 41-74.

Doria, R. B., \& Cardona, M. (2015). Turismo comunitario, como estrategia para el empoderamiento comunitario en las localidades de Ciudad Bolívar, Usme y Sumapaz, zona rural. Bogotá DC. Revista Teoría y Praxis Investigativa, 9(2), 94-114.

Esteban, N. R. R., \& Bonilla, J. (2017). Turismo y Posconflicto en el municipio de la Macarena, Meta-Colombia. RITUR-Revista Iberoamericana de Turismo, 7(1), 114134. 
Fonseca, V., Contreras, L., Porras, L., \& Prieto, A. V. (2017). Estado del arte sobre el desarrollo rural durante el periodo comprendido entre 2004-2014 en Colombia. Revista CIFE: Lecturas de Economía Social, 19(30), 121-148.

Ibáñez, A., \&Rodriguez, C. (2014). Costos económicos y sociales del conflicto en Colombia ¿Cómo construir un posconflicto sostenible? Bogotá, D.C.: Kimpres Ltda.

Ibáñez, A. M. (2016). El proceso de paz con las Farc:¿ Una oportunidad para reducir la pobreza rural y aumentar la productividad agropecuaria?. Revista de Ingeniería, (44), $8-13$.

Guilland, M. L., \& Ojeda, D. (2012). Indígenas "auténticos" y campesinos "verdes". Los imperativos identitarios del turismo en Colombia. Cahiers des Amériques latines, (71).

Guzmán Hernández, C., Garduño Mendoza, M., \&Zizumbo Villarreal, L. (2009). Reflexión crítica sobre el consumo turístico. Estudios y perspectivas en turismo, 18(6), 691-706.

Guzmán, T. J. L. G., \& Cañizares, S. M. S. (2009). Turismo comunitario y generación de riqueza en países en vías de desarrollo. un estudio de caso en El Salvador1. revesco: Revista de estudios cooperativos, (99), 85.

Inostroza, G. (2018). Aportes para un modelo de gestión sostenible del turismo comunitario en la región andina. Gestión Turística, (10), 77-90.

Laguna Arias, D. (2006). El espacio del turismo .Alteridades, 119-129.

Leiper, N. (1990). Tourist attraction systems. Annals of tourism research, 17(3), 367-384

Loewy, T. (2016). Ruralidad y desarrollo en Argentina. Estudios Rurales, 5(9).

Marzo-Navarro, M., Pedraja-Iglesias, M., \&Vinzón, L. (2017). Key variables for developing integrated rural tourism. TourismGeographies, 19(4), 575-594.

Massolo, A. (2006). El desarrollo local en la perspectiva de género. Agricultura, Sociedad y Desarrollo, Vol. 3, No. 1. Pp. 1-18.

Mora Rivera, José Jorge and Ceron Monroy, Hazael. Diversificación de ingresos en el sector rural y su impacto en la eficiencia: evidencia para México. Cuad. Desarro. Rural [online]. 2015, vol.12, n.76

Pérez, E. (2001). Hacia una nueva visión de lo rural. CLACSO, Consejo Latinoamericano de Ciencias Sociales. Obtenido de http://bibliotecavirtual.clacso.org.ar/clacso/gt/20100929011414/2perez.pdf

Orgaz Agüera, F. (2013). El turismo comunitario como herramienta para el desarrollo sostenible de destinos subdesarrollados. Nómadas, (38).

Robertt, P., \&Lisdero, P. (2016). Epistemología y metodología de la investigación sociológica: reflexiones críticas de nuestras prácticas de investigación. Sociologias, 18(41).

Roux, F. (2013). Turismo comunitario ecuatoriano, conservación ambiental y defensa de los territorios. Federación Plurinacional de Turismo Comunitario del Ecuador-FEPTCE. 
Ruiz, E., Hernández, M., Coca, A., Cantero, P., \& Campo, A. D. (2008). Turismo comunitario en Ecuador. Comprendiendo el community-basedtourism desde la comunidad. Pasos. Revista de turismo y patrimonio cultural, 6(3).

Sánchez, M. M. (2018). Colombia en posconflicto:¿ turismo para la paz o paz para el turismo?. Araucaria, 20(39).

Skewes, J. C., Zuñiga, C. H., \& Vera, M. P. (2015). Turismo comunitario o de base comunitaria: una experiencia alternativa de hospitalidad vivida en el mundo Mapuche. Tralcao sur de Chile. CULTUR-Revista de Cultura e Turismo, 6(2), 73-85.

Suriya, K. (2010, July). Impact of community-based tourism in a village economy in Thailand: An analysis with VCGE model. In EcoMod2010 conference, Istanbul, Turkey. Retrieved May(Vol. 1, p. 2013).

Vargas, M. V. R., Ocaña, Á. F. O., \&Chaviano, E. L. M. (2017). Modelo para la gestión integrada del turismo comunitario en Ecuador, caso de estudio Pastaza. REVESCO: Revista de estudios cooperativos, (123), 250-275.

Vera, L. E. H., Paladines, G. V., \& Velasco, J. E. S. (2017). La profesionalidad y humanización en trabajadores del turismo. Habilidades para el autocuidado. Revista Publicando, 4(12 (1)), 429-440 